\title{
Software defined Internet of vehicles: architecture, challenges and solutions
}

\author{
CHEN Jiacheng ${ }^{1,2}$, ZHOU Haibo ${ }^{2}$, ZHANG Ning ${ }^{2}$, YANG Peng ${ }^{2,3}$, GUI Lin ${ }^{1}$, SHEN Xuemin (Sherman) ${ }^{2}$ \\ 1. Department of Electrical Engineering, Shanghai Jiao Tong University, Shanghai 200240, China \\ 2. Department of Electrical and Computer Engineering, University of Waterloo, Waterloo, ON N2L 3G1, Canada \\ 3. Department of Information Engineering, Huazhong University of Science and Technology, Wuhan 430074, China
}

\begin{abstract}
IoV (Internet of Vehicles) is a promising paradigm to the future of automobiles, which will undoubtedly boost the automobile market as well as accelerate innovation in Internet services and applications. The concept of SD-IoV (Software Defined IoV) is presented, which is capable of improving resource utilization, service quality, and network optimization in the harsh vehicular network environments. First, A generalized SD-IoV architecture as an intuitive big picture is presented. Then, the major functions realized by SD-IoV are elabrated on to illustrate how the current challenges are resolved. As the key enablers of SD-IoV, three possible implementation methods of the wireless control path are described and compared. Finally, the challenges and existing solutions of SD-IoV are disuessed and open issues are pointed out so as to shed light on future research.
\end{abstract}

Key words: software defined networking, Internet of vehicles, network architecture, heterogeneous network, resource utilization efficiency, vehicle-to-infrastructure, vehicle-to-vehicle.

\section{Introduction}

It is envisioned that we are going to witness the commencement of a revolutionary era, in which the world will be full of intelligent objects. Specifically, owing to the unprecedented development of enabling technologies in IT(Information Technology), smart gadgets involved in our daily life are not only endowed with computation capabilities via both embedded chips and remote clouds, but also empowered with communication capabilities through wireless and backbone networks. Widely-known by a more common name-IoT (Internet of Things) ${ }^{[1]}$, such a new paradigm will dramatically enhance our future lifestyles. As an indispensable member as well as a forerunner of the huge IoT family, IoV (Internet of Vehicles) ${ }^{[2]}$ has not only appealed a lot to the automobile and information technology communities, but also gained technology maturity and public acknowledgement.

In a typical IoV (a.k.a. VCNs (Vehicular Communication Networks)) scenario, vehicles equipped with dedicated OBUs(On-Board Units) are capable of communicating with adjacent peer vehicles, forming V2V (Vehicle-to-Vehicle) connections, and receiving data services from infrastructures (e.g., RSUs (Road- 
side Units), cellular BSs (Base Stations), and Wi-Fi APs (Access Points)), all of which are regarded as V2I (Vehicle-to-Infrastructure) communications. It is believed that IoV is the cornerstone of the ITS (Intelligent Transportation System ${ }^{[3]}$ blueprint, which is envisioned to offer a variety of services ranging from road safety to mobile Internet access. For example, in case of a traffic accident, the information will be disseminated to all the vehicles in proximity via $\mathrm{V} 2 \mathrm{~V}$ communications and detour routes will be provided to the drivers who are driving towards the site. Additionally, by aggregating and analyzing real-time traffic data gathered from vehicles and sensors deployed alongside the roads, the travel planner application residing in cloud can make optimal decisions for each driver who requests the service. Ultimately, as the name IoV itself indicates, drivers and passengers in vehicles can enjoy all the services provided through Internet. Based on all these applications, the future market of IoV is deemed to be prosperous, an assumption that is further supported by a report from Gartner forecasting that there will be more than 250 million connected vehicles on the road worldwide by $2020^{[4]}$. Apart from the tremendous market demands, researches on IoV (e.g., performance analysis ${ }^{[5]}$, data dissemination $^{[6]}$, mobility analysis ${ }^{[7]}$ ) can also shed light on the development of more generalized IoT.

Despite the efforts and achievements made on VCNs in recent years, there still exist many challenges that have not been perfectly tackled yet. The first challenge is identifying how to efficiently exploit the heterogeneity of $\mathrm{VCNs}^{[8]}$. Like $5 \mathrm{G}^{[9]}$, future vehicular networking is generally expected to be heterogeneous in terms of network resources and network topology. Current cellular solutions (i.e., LTE and 4G) can almost be directly adopted by the $\mathrm{VCNs}^{[10]}$; WLANs (Wireless Local Area Networks) including both WiFi and DSRC (Dedicated Short Range Communications) interfaces only need a few adjustments so as to be utilized ${ }^{[11]}$; and even DSA (Dynamic Spectrum
Access) technologies over TVWS (TV White Space) have the potentials to be used in this field ${ }^{[12]}$. All these resources have their own strengths and advantages in the VCN scenario. For example, cellular networks have the widest coverage area and the most reliable connection, but their capacity is limited due to the ever-increasing traffic load. WLANs can offer high data delivery speed, whereas their availability is highly dependent on local infrastructures with limited coverage. The transmission characteristics of TVWS spectrum is superior and suitable for the VCN scenario. Unfortunately, the required resources are opportunistic and location-based, therefore consistent services cannot be well supported. On the other hand, two basic transmission modes, i.e., V2I and V2V, as well as other extensions co-exist in VCNs. V2I can provide Internet access to vehicles while $\mathrm{V} 2 \mathrm{~V}$ can be used for direct information dissemination, similar to D2D (Device-to-Device) communications in LTE networks. Definitely, the heterogeneity mentioned above poses tough challenges for high resource utilization efficiency and capacity boost.

Second, it is of significant challenge to meet the diverse requirement of QoS (Quality of Service) in VCNs. Future connected vehicles are expected to play an important role as mobile centers of information gathering and processing in day-to-day human life. Therefore, IoV should be able to accommodate differentiated services requests with different QoS requirements. For example, safety-related services require low latency and high reliability, streaming services exert strict constraints on connection speed and stability, and delay-tolerant services are generally bandwidth-consuming. However, heterogeneous network substrates can hardly support the distinction of these services, and the network does not have a global view of all the service requests in order to make compromises among them for the sake of serving as many requests as possible.

The third challenge is how to manage and control the VCNs in a scalable and flexible manner. With the 
ever-increasing vehicular network size and density as well as the highly-evolved physical layer technology, the management and control of VCNs will become an underlying bottleneck impeding vehicular network performance. That is, due to the high mobility of vehicles, handoff events among various infrastructures are more frequent, compared with traditional wireless networks. Passive handoff without prior knowledge on vehicles' traces is inefficient and is likely to cause service interruption. Also, access and admission control should be tightly coupled with the QoS-driven resource allocation process. However, there is still a lack of unified and universal ways to deal with the management and control issues that arise in heterogeneous VCNs.

In order to solve the aforementioned challenges, a consensus has been gradually reached on the necessity of a fundamental reconsideration on the IoV design. Among all the candidates, SD-IoV (Software Defined IoV) has successfully demonstrated its superiorities against others. As the name suggests, it is the integration of SDN (Software Defined Networking) ${ }^{[13]}$ and IoV. SDN is an emerging networking paradigm mainly differentiated by the separation of control and data plane. The control plane consists of centralized controllers, which dynamically install forwarding rules into the switches in the data plane. SDN is known to be able to bring programmability and flexibility to the current networks and has been adopted in various kinds of scenarios including wired datacenter networks ${ }^{[14]}$, wireless mesh networks ${ }^{[15]}$, WLANs ${ }^{[16]}$, narrow sense IoT $^{[17]}$, wireless sensor networks ${ }^{[18]}$, cellular networks ${ }^{[19]}$, etc., either conceptually, experimentally, or even practically. Tab.1 briefly summarizes the progress of SDN implementation in these networks. However, due to the unique characteristics of VCN such as high mobility and heterogeneity, SDIoV study is still in its infancy. In this paper, we aim to offer a deep understanding on the significance of SD-IoV and gather people's attention on this topic. In the remaining of this paper, we first provide a generalized architecture of SD-IoV and introduce its necessary components. A layered architecture is also provided so as to show the SD-IoV system from a different view. Following this, key functions enabled by SD-IoV are described, which serve as the exemplifications on how the challenges in current $\mathrm{VCNs}$ can be solved. As a critical step towards realizing SD-IoV, three possible implementation methods of wireless control path are presented and compared. In order to pave the way for future researches, the challenges of SD-IoV as well as existing solutions are summarized, followed by open issues. Finally, we present the conclusion of this paper.

Table 1

Summary of SDN implementation progress in different types of networks

\begin{tabular}{|c|c|c|c|}
\hline & $\begin{array}{l}\text { critical issues to be considered during } \\
\text { SDN implementation }\end{array}$ & $\begin{array}{l}\text { key benefits of } \\
\text { enabling SDN }\end{array}$ & $\begin{array}{l}\text { SDN implementation } \\
\text { progress }\end{array}$ \\
\hline $\mathrm{IoV}^{[20-25]}$ & $\begin{array}{l}\text { high end-node mobility, resource } \\
\text { heterogeneity }\end{array}$ & $\begin{array}{l}\text { high resource utilization efficiency, } \\
\text { easy mobility management }\end{array}$ & infancy \\
\hline IoT (narrow sense) ${ }^{[17]}$ & device heterogeneity & unified management & prior research \\
\hline WSN (Wireless Sensor Network) ${ }^{[18]}$ & energy-constrained nodes & network lifetime extension, sensor reuse & prior research \\
\hline WMN (Wireless Mesh Network) ${ }^{[15]}$ & $\begin{array}{l}\text { spectrum scarcity, mesh router } \\
\text { hardware limitation }\end{array}$ & $\begin{array}{l}\text { global-view routing and } \\
\text { resource allocation }\end{array}$ & prior research \\
\hline cellular network $^{[19]}$ & control functions offloading and distribution & $\begin{array}{l}\text { energy saving, interference reduction, } \\
\text { CoMP (Coordinated Multi-Point) }\end{array}$ & progressive research \\
\hline WLAN $^{[16]}$ & end-user compatibility & load balancing, seamless handoff & prototyping \\
\hline wired network $^{[14]}$ & non specific & $\begin{array}{l}\text { flexibility of network adjustment, } \\
\text { switch simplicity, virtualization, etc. }\end{array}$ & practical application \\
\hline
\end{tabular}




\section{Software defined loV: architecture and functions}

\subsection{Architecture}

We introduce a generalized and intuitive architecture of SD-IoV, as shown in Fig.1. It aims to highlight the backbone components and their major functionalities, which are described as follows:

- Logical SDN controllers. SDN controllers are basically software programmes running in servers and have a global view of all the other components in the SD-IoV system. As the core components of SD-IoV, they are responsible for not only network management and operation (e.g., rule generation, network virtualization, client association, resource allocation, and mobility management) but also some advanced functionalities (e.g., data pre-processing, network analysis, and learning). The word "logical" can be interpreted from two aspects. On one hand, it indicates that the SDN controllers can physically be placed in cloud, and/or in the local areas (for scalability and delay-reduction purposes). On the other hand, all these functionalities can be distributed among different controllers in a hierarchical manner.

- SDN switch network. We suppose that the SDN switch network is operated by the same independent SD-IoV operator and is connected to the core Internet via high-speed links. However, this does not necessarily mean that SDN switch network is localized or scale-limited. Actually, similar to Google's software defined WAN (Wide Area Network), it can expand as large as a global network, depending on the operator's demand. Consider a download session as an illustrative example. When packets from the servers in the Internet arrive at the SDN switch network, they are for- warded to the SDN-enabled switches according to the pre-installed rules. If there is no matching rule, then the packets are forwarded to the SDN controller and thereafter forwarded to the appropriate switches according to the decision made by the controller, just after the rule installation directions are delivered to the corresponding switches. In this part, the packets are finally routed to the wireless access infrastructures that serve the requesting vehicles.

- SDN-enabled wireless access infrastructures. For a heterogeneous IoV, infrastructures can be Wi-Fi APs, cellular BSs, RSUs, or even DSA coordinators. All of them work for providing wireless interfaces (a.k.a. last mile accesses) to the associated vehicles. In the above example, the final step of the download process is conducted by transmitting the packets to the requesting vehicles. Also, these infrastructures are SDN-enabled, meaning that the SDN controller can control their behavior, at least to some extent. Such behavior may include general SDN functionalities such as packet forwarding and transmitting, as well as operations specific to the infrastructures such as power control for the BSs, operation channel assignment for the APs, and resource allocation for the DSA coordinators.

- SDN-enabled vehicles (OBUs). Vehicles are generally the end-users in the SD-IoV system. In the $\mathrm{V} 2 \mathrm{~V}$ cases, they also act as relays. We consider that OBUs have multi-homing capabilities and enable a certain part of full SDN supports, based on the OBU designs and implementations. In other words, the hardware support of OBUs decides to what extent the SDN controllers can control them. The potential SDN-enabled functions of OBUs include but not are limited to: packet forwarding, power control, channel selection, interface selection, and transmission mode selection. 


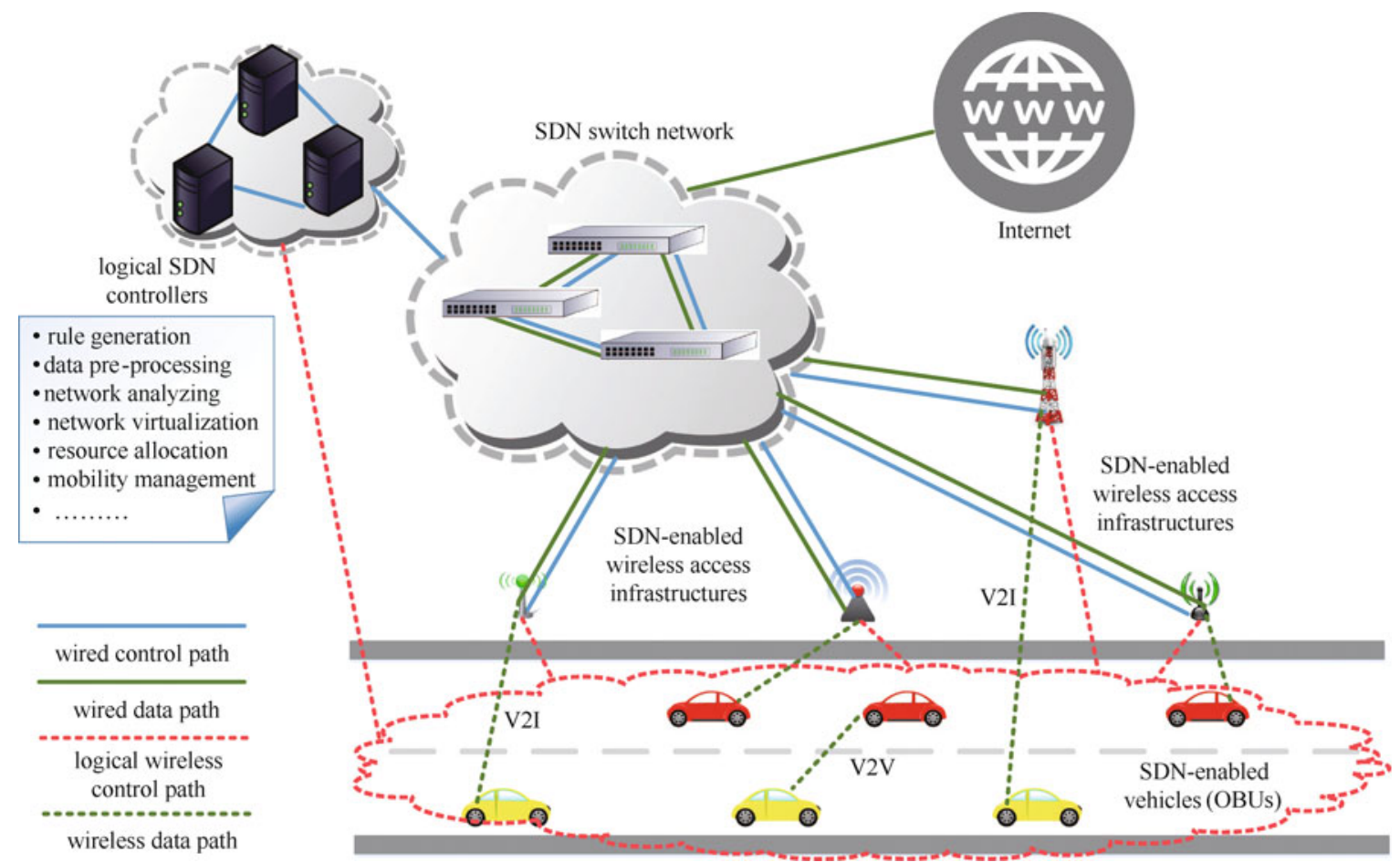

Figure 1 Architecture of software defined IoV

- Data path. Data path comprises both wired and wireless links. Wired links are used for data transmission between the Internet, SDN switch networks, and SDN-enabled wireless access infrastructures. Wireless links provide data exchange services for the SDN-enabled vehicles. Note that for the wireless data paths, various types of technologies can be used, depending on the infrastructures. Also, no changes are required at the lower layers (i.e., PHY and MAC).

- Control path. The main responsibilities of control paths are to: 1) ensure that the other components in SD-IoV can be controlled by the controllers; 2) guarantee real-time state feedbacks from other components. Wired control paths are used among SDN controllers, SDN switch networks, and SDN-enabled wireless access infrastructures, just the same as normal SDNs. However, wireless control paths can be implemented in different ways, with different requirements on control channels, protocols and hardware supports. Hence, we only use logical wireless control paths to link the vehicles and controllers in the illustrative architecture. In Fig.2, we present a layered architecture of SDIoV so as to better illustrate it from a different angle. From top to bottom, these planes are introduced below.

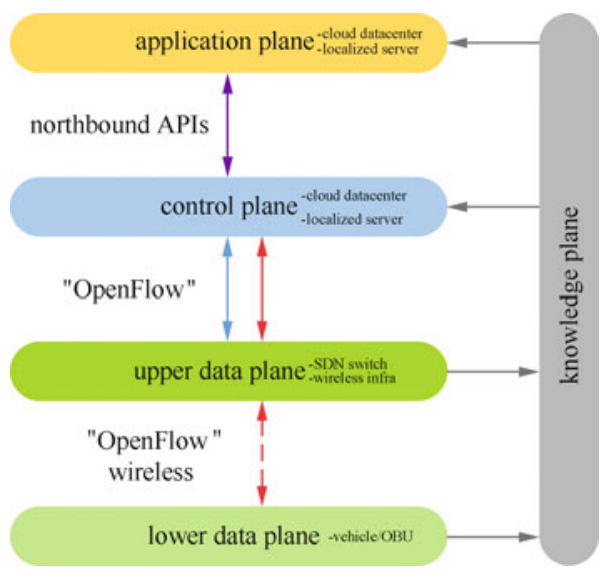

Figure 2 Layered architecture of software defined IoV

- Application plane and control plane. Both the 
application plane and control plane physically reside in cloud datacenters and localized servers. Application plane accommodates all the network services on the top of controllers in the control plane, which provides APIs to realize the services and translate services into rules. In other words, the network services are just the software programmes to implement the controller functionalities described above. The relationship between application and control planes can be compared to the relationship between softwares and operating systems in computers.

- Upper data plane. The upper data plane corresponds to the SDN switches and SDN-enabled wireless access infrastructures. It is the physical carrier for packet transmission in wired networks, so it can also be called as wired data plane. The rules are set by the control plane through an "enhanced" OpenFlow protocol ${ }^{[26]}$. Specifically, due to the involvement of wireless access infrastructures, extension of OpenFlow is preferred so as to realize more advanced functionalities than simple packet forwarding, such as power control of cellular BSs and activation of power-saving mode of Wi-Fi APs.

- Lower data plane. The lower data plane is composed of SDN-enabled vehicles, which are end-users and sometimes act as relays (i.e., in $\mathrm{V} 2 \mathrm{~V}$ communications). It is also equivalent to the wireless data plane or the user plane. A wireless extension of OpenFlow protocol is required such that the commands from the control plane can be understood. Like upper data plane, the extensions are specifically designed for the configuration of OBUs, such as vertical handoff among different wireless interfaces.

- Knowledge plane. Knowledge plane is an abstrac- tion of the network state feedback functionalities. In order to generate appropriate rules, the application plane and control plane need necessary information about the current states of data planes. However, it need not be the same as the original information, which means that some modifications can be done in this plane for various purposes, such as data size reduction and privacy preservation.

\subsection{SD-IoV functions}

In this subsection, we will exemplify how functions enabled by SD-IoV solves the aforementioned challenges in traditional VCNs. Note that the benefits of traditional SDN also apply to the SD-IoV scenario, such as easy application deployment, vendor neutralization, network management flexibility, etc. Meanwhile, we will progressively present three possible wireless control path implementation methods (here we focus on the control functionality), which are the critical steps towards extending SDN to wireless, and specifically IoV scenarios.

\subsubsection{Vehicular packet transmission control}

Packet transmission control is the fundamental application of SD-IoV, yet it is the best way to demonstrate how SD-IoV can achieve interoperability in a heterogeneous scenario and can further efficiently utilize the heterogeneous resources. Packet transmission control in the wired network is the same as normal SDNs. However, in the wireless part, it strongly relies on the implementation of wireless control path. For simplifying the implementation, we assume that the packet transmission works in the same way as normal after the association between infrastructures and vehicles has been done. Hence, we mainly discuss how to control the wireless interface that a transmission will use. Three levels of wireless control path implementation methods with different requirements on resources 


\begin{tabular}{|c|c|c|c|c|}
\hline \multirow{2}{*}{ features } & \multicolumn{3}{|c|}{ wireless control path implementation (SD-IoV) } & \multirow{2}{*}{ traditional IoV } \\
\hline & none & piggyback & dedicated & \\
\hline software defined V2I download & straightforward & straightforward & straightforward & not supported \\
\hline software defined V2I upload & trial and error & handshake & straightforward & not supported \\
\hline software defined V2V & not supported & handshake & straightforward & not supported \\
\hline OBU hardware support for SDN & not required & slight & Heavy & not required \\
\hline wireless OpenFlow extension & not required & slight & Heavy & not required \\
\hline cost & low & medium & high & none \\
\hline delay & large & medium & small & small \\
\hline resource utilization efficiency & medium & medium & high & low \\
\hline
\end{tabular}

and supports will be presented in the following. Fig.3 shows simple illustrations of the implementation procedures of the methods. Also, the main differences among these methods are summarized in Tab.2.

First, we suppose that there is no wireless control path, i.e., SDN is only enabled in the wired networks. In the download case (Fig.3(a)), the packet is routed to a certain SDN-enabled wireless access infrastructure, based on the resource-utilization-optimized decision made by the controller. The infrastructure thereafter adds the destination address in the packet header and sends it out. Due to the multi-homing capability, the OBU with the same destination address will receive the packet and continue on further processing. In the upload case (Fig.3(b)), the OBU arbitrarily selects an interface and sends its packet. Upon acknowledgment of the request, the controller decides the optimal interface for the flow. If the interface is not consistent with the one that OBU was using, then the OBU will not get any response. Once time-out, the OBU knows that it had selected a wrong interface and it will pick another one for the next attempt. We name the above interaction process as trial and error. Obviously, in this scenario, requirement on system design is the simplest since neither SDN-enabled
OBU nor wireless OpenFlow extension is required. However, this is not a completely software defined IoV and can lead to several drawbacks, which are listed in Tab.2. Some of the drawbacks are no software defined V2V communications support, large delay, and medium resource utilization efficiency.

Second, we suppose that the wireless control path is implemented through piggyback within each individual network. In other words, each network uses its own resource to manage the control of vehicles. The download case is straightforward as it is in the first method. For the upload case (Fig.3(c)), a handshake is required to determine which wireless interface should be used. Specifically, an OBU sends the upload request through an arbitrary interface. Then, the response is sent via a certain interface, which is decided by the controller and will be used for further data transmission. Upon receiving the response, the OBU knows the interface assigned by the controller. Also, V2V communications can be enabled in a similar manner (Fig.3(d)). After the initiating vehicle sends the request with an arbitrary interface, the controller decides both the transmitting vehicle and the interface, through which the controller then informs both vehicles. However, this method requires minimal 

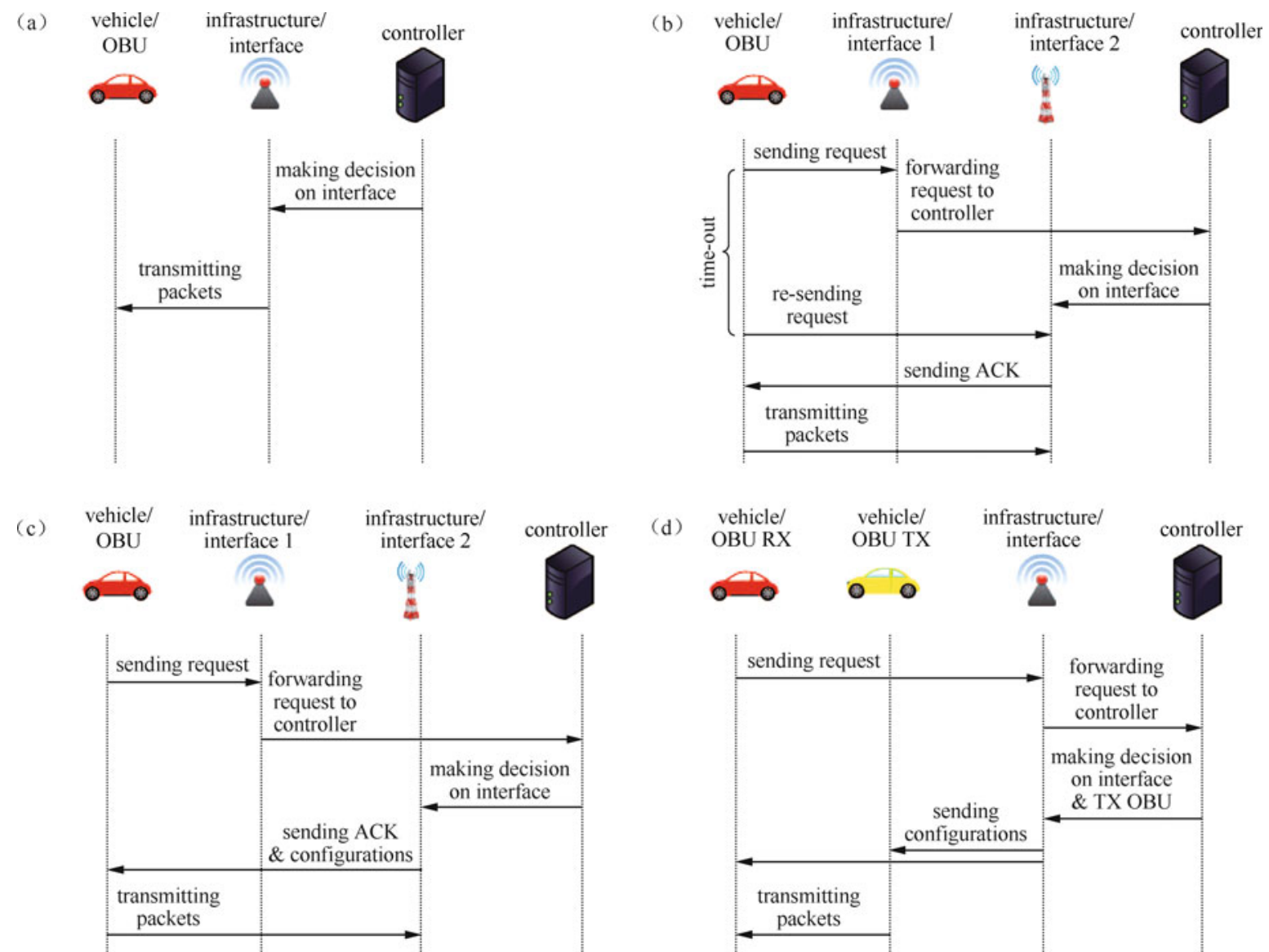

Figure 3 Procedures of wireless control path implementation methods: (a) straightforward download; (b) trial and error upload; (c) handshake upload; (d) handshake V2V

OBU's support for SDN as well as wireless extension of OpenFlow so as to realize the handshake process. The advantages are, V2V can be supported and delay can be reduced to some extent.

Last, we suppose that there exists a dedicated channel for the wireless data path. In this way, regardless of whichever case, packet transmission will be straightforward. Moreover, by upgrading the OBU hardware supports and extensions of OpenFlow, more advanced functionalities on the vehicle side can be realized, e.g., configuration of SDR (Software Defined Radio) operations in the OBU. In this way, the resource utilization efficiency can be further improved. However, such significant changes will lead to high development cost and deployment difficulties.

\subsubsection{Vehicular network virtualization}

SDN is known to be intrinsically suitable for realizing network virtualization, and the SD-IoV is by no means an exception. Specifically, through the regulation of traffic flow and configuration of wireless access infrastructures as well as vehicles, network resources can be easily sliced and clearly isolated, which is the so-called network virtualization. From the perspective of a specific user/service, it is as if it owns the entire network. Originally, the aim of 
network virtualization is to improve resource utilization efficiency since the physical infrastructures (e.g., switches) can be shared by different users. However, it can also be used to guarantee the QoS of high-priority services in IoV, by constructing a virtualized network with dedicated resources for the services.

\subsubsection{Vehicular access handoff}

In traditional wireless networks, the handoff process is passively activated when mobile users cross the intersection area of adjacent cells, which is also the main reason for performance loss. However, in SDIoV, since the controller has a global view, the handoff process can be prepared prior to the real handoff time. Also, the handoff process itself can be dramatically simplified. Specifically, for each service/vehicle, a virtualized agent can be generated, which retains the necessary information, such as destination IP address, to maintain the connection. During a handoff, the controller just migrates the virtualized agent from the original infrastructure to the new one, and simultaneously allocates network resources and informs the handoff vehicle to prepare for actions such as interface change.

\section{Software defined loV: challenges and solutions}

\subsection{Flexible and scalable resource schedul- ing}

In SD-IoV, heterogeneous resources can be centrally managed by the controllers, thus it is possible that global optimality of resource utilization efficiency can be achieved and QoS of critical services can be guaranteed. However, the design of such resource scheduling algorithms with flexibility and scalability is a big challenge, due to 1) the heterogeneity of resource types and connection modes; and 2) the computation complexity and scalability issues caused by centralization. In Ref.[20], the authors aim to schedule different network resources to minimize communication cost. A network availability vector as well as a network cost vector are constructed first, and the scheduling is formulated as an optimization problem subject to bandwidth constraint. Exhaustive search and greedy algorithms are presented to solve the problem. In another work ${ }^{[21]}$, Liu, et al. study the CDS (Cooperative Data Scheduling) problem with the objective of maximizing weighted gain. Each vehicle has a weight, which is inversely proportional to the estimated remaining dwell time. The scheduling period is divided into three phases: V2V, V2I and hybrid; and the centralized controller in the RSU will decide which vehicle participates in V2I or V2V in the third phase. The CDS problem is solved through an online scheduling algorithm based on a greedy method.

\subsection{Control functionality deployment}

Control functionality deployment is a generalization of issues related to control, including the placement of control functionalities, both physically and logically; and the implementation of control paths. In traditional SDN, the problem of controller placement has been a major issue. On one hand, it is argued that some of the controllers should be placed near the network edge so as to reduce delay. On the other hand, from the perspective of control functionality, there are also some researchers advocating a hierarchical architecture, in which some of the functionalities are distributed to localized controllers whereas the central/high-level controller only needs to guide these lower-level controllers. In SD-IoV, the control functionality deployment becomes more complicated since wireless access infrastructures and vehicles are incorporated in the SDN management domain. In Section 2.2, we 
have proposed three SDN control implementation methods with different levels of requirements on system hardware and software supports. Basically, the challenge is to find an optimal compromise between implementation complexity, which directly influences technology penetration speed and economic benefits, and the capability of SD-IoV system. In Ref.[22], $\mathrm{Ku}$, et al. use LTE/WiMAX for control plane and Wi-Fi for data plane. They also assume a local agent in the wireless node, which makes it controllable. Three operational modes are proposed: central control mode, in which the SDN controller controls all actions; distributed control mode, in which only the wireless nodes control themselves via local agents; and hybrid control mode, in which the SDN controller sends general policy rules instead of concrete flow rules to the RSUs to define general behaviors, based on which RSUs use local intelligence to make further forwarding decisions. A similar idea is presented by Truong, et al. in Ref.[23]. The authors leverage the concept of fog computing and design an architecture called FSDN VANET. As its name indicates, fog lies between cloud and ground, and it is basically equivalent to a near-sighted controller. Specifically in FSDN VANET, the fog is composed of RSU, RSU controller and BS, and the SDN controller is responsible for fog orchestration and network management.

\subsection{SDN operation optimization}

SDN operation refers to the behaviors specific to SDN/OpenFlow, such as rule generation and installation, and VM (Virtual Machine) migration. Optimization of these operations will definitely increase the efficiency and robustness of SDN. However, the introduction of wireless data plane, which is essentially the incorporation of end-users into SDN, differentiates SD-IoV from traditional SDN. New challenges will arise due to the growing density of software defined vehicles, high mobility, wireless channel state dynamics, etc. In Ref.[24], the authors identify the reconfiguration cost problem in RSU clouds, which consist of the cloud controller, OpenFlow controller, cloud resource manager, and traditional RSU. Reconfiguration is triggered simultaneously with handoff events, network state changes, etc., and its overheads mainly come from VM migration among physical infrastructures and control plane (rule) modifications. The authors perform a real-world reconfiguration overhead analysis, and formulate the RSU CRM (Cloud Resource Management) problem as an ILP (Integer Linear Programming). Since the problem has multiple objectives, the Pareto optimal frontier is solved first and thereafter a heuristic algorithm is developed to minimize the reconfiguration cost. In Ref.[25], Wang, et al. consider the limited size of flow tables in OpenFlow switches and aim to build more compact rules to ensure scalability. A rule optimization approach is developed by using multicast address in the wireless data plane. Furthermore, a destination-driven model is introduced in the wired data plane so as to reduce the rule items in switches.

\section{Software defined loV: open issues}

\subsection{Resource abstraction}

As mentioned before, the heterogeneity of SD-IoV greatly increases the complexity and intractability of fine-grained resource allocation. One potential way to alleviate this problem is the abstraction of physical resources. In other words, if the heterogeneous resources can be described in a uniform language, the formulation of the resource scheduling problem will be much clearer and the programmability offered by SDN will be utilized more efficiently. In computer sciences, the concept of resource virtualization has been acknowledged and implemented almost since the beginning. For example, the CPU resources are 
abstracted as time chips to be used by processes. Also, in wireless communications, resource abstraction is achieved with the introduction of multiplexing, e.g., TDM (Time Division Multiplexing). However, the resources in the above mentioned scenarios are homogeneous. Recently, a notable progress towards resource abstraction is accomplished in LTE/4G networks, i.e., OFDMA (Orthogonal Frequency-Division Multiple Access) technology, which uses resource block as the basic resource element. Nevertheless, such abstraction is still limited to a single network, and its extension to the heterogeneous SD-IoV may not be applicable. A possible primer solution is to build an abstraction layer between the wireless access infrastructures and the controller and define the corresponding resource translation protocol.

Furthermore, the evaluation method of the abstracted resources should also be studied, in order to face the service diversity and harsh environment of IoV scenario. As mentioned before, different resources with various characteristics have their advantages and applicable scenarios. The evaluation method should be multi-dimensional so as to reflect these characteristics, and more importantly shed light on the optimal allocation of these resources. surrounding information updating.

\subsection{Network intelligence}

$\mathrm{SD}-\mathrm{IoV}$ is inherently appropriate for realizing an intelligent network, which means the controller has the ability to dynamically optimize the network resource management through learning-based algorithms. We will use two examples to illustrate this feature in detail. In the first example, a general cognition cycle can be built to describe the process. The controller not only gathers real-time network state information but also uses it as a stamp for the storage of other data such as resource utilization index and QoS satisfaction index. After a pre-processing phase, e.g., anonymization, all the gathered data are used as the training datasets for certain machine learning algorithms. When the algorithms are well-tuned and can outperform the existing resource allocation algorithms (usually heuristic algorithms due to computation complexity), they can be adopted by the real SDIoV, taking the real-time network state as input and outputting the resource scheduling decisions. In the second example, the learning methods are used for prediction. Specifically, the vehicle traffic states (i.e., the network density and mobility states) and the data traffic states are mined from historical data and exploited for predictions. In the IoV scenario, such state information is highly regular due to the strong correlation with contexts, such as time and location. For example, more vehicles are on the road during rush hours and vehicles tend to request more data near PoIs (Point of Interests). Therefore, predictions can be done with relatively high accuracy and are useful for the controller to make better decisions.

\subsection{Security and privacy}

As a centrally controlled and location-based system, SD-IoV will encounter various security and privacy challenges. In the control plane, the controller requires circumstantial descriptions on the status of all vehicles, such as current locations and destinations. If the controller cannot be trusted or its databases are breached, it will cause severe privacy issues of vehicular end-users. Also, due to centralization, SDIoV is vulnerable to DoS (Denial-of-Service) attacks. The control functionality may be paralyzed by a huge number of fake requests received within a short time window. Moreover, if the controller itself is seized by unauthorized people, the whole network may come under the control of the attacker, who can then utilize it for further malicious purposes such as initiating distributed DoS attacks to other networks or valuable data theft. 


\section{Conclusion}

In this paper, we identified the primary challenges of current VCNs and introduced the SD-IoV. First, we presented a generalized architecture of SD-IoV, followed by a layered architecture. We then established the benefits of SD-IoV through three key functions. Furthermore, we proposed three implementation methods of wireless control path and compared their functionalities and performance. We summarized the challenges of SD-IoV and their existing solutions. Finally, we shared our view on the open issues in the field of SD-IoV.

\section{References}

[1] AL-FUQAHA A, GUIZANI M, MOHAMMADI M, et al. Internet of things: a survey on enabling technologies, protocols, and applications[J]. IEEE communications surveys \& tutorials, 2015, 17(4): 2347-2376.

[2] LU N, CHENG N, ZHANG N, et al. Connected vehicles: solutions and challenges[J]. IEEE Internet of Things journal, 2014, 1(4): 289-299.

[3] PAPADIMITRATOS P, LA FORTELLE A, EVENSSEN K, et al. Vehicular communication systems: enabling technologies, applications, and future outlook on intelligent transportation[J]. IEEE communications magazine, 2009, 47(11): 84-95.

[4] Velosa A, HINES J F, LEHONG H, et al. Predicts 2015: the internet of things [EB/OL]. https://www.gartner.com/doc/2952822/ predicts-internet-things.

[5] LU N, ZHANG N, CHENG N, et al. Vehicles meet infrastructure: toward capacity-cost tradeoffs for vehicular access networks[J]. IEEE transactions on intelligent transportation systems, 2013, 14(3): 1266-1277.

[6] ZHOU H, LIU B, LUAN T H, et al. ChainCluster: engineering a cooperative content distribution framework for highway vehicular communications[J]. IEEE transactions on intelligent transportation systems, 2014, 15(6): 2644-2657.

[7] WANG M, SHAN H, LUAN T H, et al. Asymptotic throughput capacity analysis of VANETs exploiting mobility diversity[J]. IEEE transactions on vehicular technology, 2015, 64(9): 4187-4202.

[8] ZHENG K, ZHENG Q, CHATZIMISIOS P, et al. Heterogeneous vehicular networking: a survey on architecture, challenges, and solutions[J]. IEEE communications surveys \& tutorials, 2015, 17(4): 2377-2396.

[9] ZHANG N, CHENG N, GAMAGE A T, et al. Cloud assisted HetNets toward $5 \mathrm{G}$ wireless networks[J]. IEEE communications magazine, 2015, 53(6): 59-65.

[10] FETEIHA M F, HASSANEIN H S. Enabling cooperative relaying vanet clouds over Lte-A networks[J]. IEEE transactions on ve- hicular technology, 2014, 64(4): 1468-1479.

[11] KENNEY J B. Dedicated short-range communications (DSRC) standards in the United States[J]. Proceedings of the IEEE, 2011, 99(7): 1162-1182.

[12] CHEN J, LIU B, ZHOU H, et al. Providing vehicular infotainment service using VHF/UHF TV bands via spatial spectrum reuse[J]. IEEE transactions on broadcasting, 2015, 61(2): 279-289.

[13] MACEDO D F, GUEDES D, VIEIRA L F M, et al. Programmable networks - from software-defined radio to software-defined networking[J]. IEEE communications surveys \& tutorials, 2015, 17(2): 1102-1125.

[14] JAIN S, KUMAR A, MANDAL S, et al. B4: experience with a globally-deployed software defined wan[C]//Proceedings of the ACM SIGCOMM 2013 conference on SIGCOMM, Hong Kong, China, c2013, 3-14.

[15] HUANG H, LI P, GUO S, et al. Software-defined wireless mesh networks: architecture and traffic orchestration[J]. IEEE network, 2015, 29(4): 24-30.

[16] SURESH L, SCHULZ-ZANDER J, MERZ R, et al. Towards programmable enterprise WLANS with $\operatorname{Odin}[\mathrm{C}] / /$ Proceedings of the 1st workshop on Hot Topics in Software Defined Networks, Helsinki, Finland, c2012, 115-120.

[17] LIU J, LI Y, CHEN M, et al. Software-defined internet of things for smart urban sensing $[\mathrm{J}]$. IEEE communications magazine, 2015, 53(9): 55-63.

[18] ZENG D, LI P, GUO S, et al. Energy minimization in multitask software-defined sensor networks[J]. IEEE transactions on computers, 2015, 64(11): 3128-3139.

[19] BRADAI A, SINGH K, AHMED T, et al. Cellular software defined networking: a framework[J]. IEEE communications magazine, 2015, 53(6): 36-43.

[20] HE Z, ZHANG D, LIANG J. Cost-efficient heterogeneous data transmission in software defined vehicular networks[C]//Proceedings of IEEE 17th International Conference on High Performance Computing and Communications (HPCC), New York, USA, c2015; 666-671.

[21] LIU K, NG J K Y, LEE V, et al. Cooperative data scheduling in hybrid vehicular ad hoc networks: VANET as a software defined network[J]. IEEE/ACM transactions on networking, 2015, pp(qq):1.

[22] KU I, LU Y, GERLA M, et al. Towards software-defined VANET: architecture and services[C]//Proceedings of the 13th Annual Mediterranean Ad Hoc Networking Workshop (MED-HOC-NET), Piran, Slovenia, c2014: 103-110.

[23] TRUONG N B, LEE G M, GHAMRI-DOUDANE Y. Software defined networking-based vehicular ad hoc network with fog computing[C]//Proceedings of IFIP/IEEE International Symposium on Integrated Network Management (IM), Ottawa, Canada, c2015: 1202-1207.

[24] SALAHUDDIN M A, AL-FUQAHA A, GUIZANI M. Softwaredefined networking for RSU clouds in support of the internet of vehicles[J]. IEEE Internet of Things journal, 2015, 2(2): 133-144.

[25] WANG X, WANG C, JIANG C, et al. Rule optimization for realtime query service in software-defined Internet of vehicles[J]. 
arXiv: 1503.05646 .

[26] MCKEOWN N, ANDERSON T, BALAKRISHNAN H, et al.
OpenFlow: enabling innovation in campus networks[J]. SIGCOMM comput. commun. rev., 2008, 38(2): 69-74.

\section{About the authors}



CHEN Jiacheng received his B.Eng. degree from the School of Electronic Information and Electrical Engineering, Dalian University of Technology, Dalian, China, in 2013. He is now pursuing his Ph.D. degree in the Department of Electronic Engineering, Shanghai Jiao Tong University, Shanghai, China. His current research interests include resource management in wireless networks, vehicular ad hoc networks and white space networks. (Email: jiacheng1989@gmail.com)

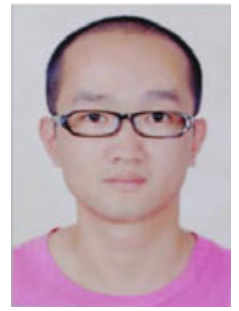

ZHOU Haibo [corresponding author] received the Ph.D. degree in Information and Communication Engineering from Shanghai Jiao Tong University, Shanghai, China, in 2014. He is currently a Post-Doctoral Fellow with the BBCR (Broadband Communications Research) Group, University of Waterloo. His current research interests include resource management and performance analysis in cognitive radio networks and vehicular networks. (Email: haibozhouuw@gmail.com)

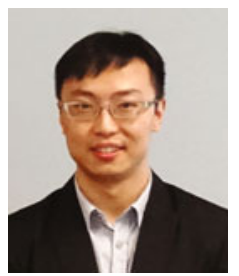

ZHANG Ning received the Ph.D. degree from University of Waterloo in 2015. He received his B.Sc. degree from Beijing Jiaotong University and the M.Sc. degree from Beijing University of Posts and Telecommunications, Beijing, China, in 2007 and 2010, respectively. From May 2015 to April 2016, he was a postdoc research fellow at BBCR lab in University of Waterloo. He is now a postdoc research fellow at University of Toronto. His current research interests include next generation wireless networks, software defined networking, green communication, and physical layer security. (Email: zhangningbupt@ gmail.com)

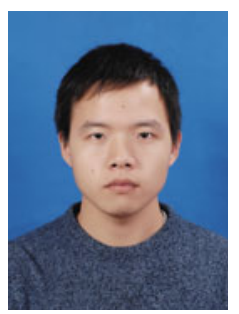

YANG Peng received his B.Sc. degree from the Department of Electronics and Information Engineering, Huazhong University of Science and Technology, Wuhan, China, in 2013. Currently, he is pursuing his $\mathrm{Ph} . \mathrm{D}$. degree in the School of Electronic Information and Communications, HUST. From Sept. 2015, he is also a visiting Ph.D. student in the Department of Electrical and Computer Engineering, University of Waterloo, Canada. His current research interests include next generation wireless networks and software-defined networking. (Email: yangpeng@hust. edu.cn)

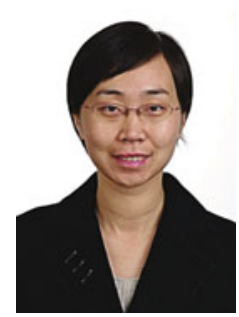

GUI Lin received the Ph.D. degree from Zhejiang University, Hangzhou, China, in 2002. Since 2002, she has been with the Institute of Wireless Communication Technology, Shanghai Jiao Tong University, Shanghai, China. Currently, She is a Professor in Shanghai Jiao Tong University. Her current research interests include HDTV and wireless communications. (Email: guilin@sistu.edu.cn)

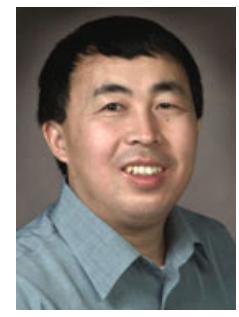

SHEN Xuemin (Sherman) received the B.Sc (1982) degree from Dalian Maritime University (China) and the M.Sc. (1987) and Ph.D. degrees (1990) from Rutgers University, New Jersey (USA), all in electrical engineering. $\mathrm{He}$ is a Professor and University Research Chair, Department of Electrical and Computer Engineering, University of Waterloo, Canada. He was the Associate Chair for Graduate Studies from 2004 to 2008. Dr. Shen's research focuses on resource management in interconnected wireless/wired networks, wireless network security, social networks, smart grid, and vehicular ad hoc and sensor networks. He is a co-author/editor of six books, and has published more than 600 papers and book chapters in wireless communications and networks, control and filtering. Dr. Shen served as the Technical Program Committee Chair/ Co-Chair for IEEE Infocom'14, IEEE VTC'10 Fall, the Symposia Chair for IEEE ICC'10, the Tutorial Chair for IEEE VTC'11 Spring and IEEE ICC'08, the Technical Program Committee Chair for IEEE Globecom'07, the General Co-Chair for Chinacom'07 and QShine'06, the Chair for IEEE Communications Society Technical Committee on Wireless Communications, and P2P Communications and Networking. He also serves/served as the Editor-in-Chief for IEEE Network, Peer-to-Peer Networking and Application, and IET Communications; a Founding Area Editor for IEEE Transactions on Wireless Communications; an Associate Editor for IEEE Transactions on Vehicular Technology, Computer Networks, and ACM/Wireless Networks, etc.; and the Guest Editor for IEEE JSAC, IEEE Wireless Communications, IEEE Communications Magazine, and ACM Mobile Networks and Applications, etc. Dr. Shen received the Excellent Graduate Supervision Award in 2006, and the Outstanding Performance Award in 2004, 2007 and 2010 from the University of Waterloo, the PREA (Premier's Research Excellence Award) in 2003 from the Province of Ontario, Canada, and the Distinguished Performance Award in 2002 and 2007 from the Faculty of Engineering, University of Waterloo. Dr. Shen is a registered Professional Engineer of Ontario, Canada, an IEEE Fellow, an Engineering Institute of Canada Fellow, a Canadian Academy of Engineering Fellow, and a Distinguished Lecturer of IEEE Vehicular Technology Society and Communications Society. (Email: sshen@uwaterloo.ca) 